\title{
Review Article \\ Biomedical and Clinical Promises of Human Pluripotent Stem Cells for Neurological Disorders
}

\author{
Nopporn Jongkamonwiwat ${ }^{1}$ and Parinya Noisa ${ }^{2}$ \\ ${ }^{1}$ Faculty of Health Sciences, Srinakharinwirot University, Ongkharak, Nakhon Nayok 26120, Thailand \\ ${ }^{2}$ School of Biotechnology, Institute of Agricultural Technology, Suranaree University of Technology, \\ Nakhon Ratchasima 30000, Thailand
}

Correspondence should be addressed to Parinya Noisa; p.noisa@sut.ac.th

Received 13 July 2013; Accepted 13 August 2013

Academic Editor: Ken-ichi Isobe

Copyright (c) 2013 N. Jongkamonwiwat and P. Noisa. This is an open access article distributed under the Creative Commons Attribution License, which permits unrestricted use, distribution, and reproduction in any medium, provided the original work is properly cited.

\begin{abstract}
Neurological disorders are characterized by the chronic and progressive loss of neuronal structures and functions. There is a variability of the onsets and causes of clinical manifestations. Cell therapy has brought a new concept to overcome brain diseases, but the advancement of this therapy is limited by the demands of specialized neurons. Human pluripotent stem cells (hPSCs) have been promised as a renewable resource for generating human neurons for both laboratory and clinical purposes. By the modulations of appropriate signalling pathways, desired neuron subtypes can be obtained, and induced pluripotent stem cells (iPSCs) provide genetically matched neurons for treating patients. These hPSC-derived neurons can also be used for disease modeling and drug screening. Since the most urgent problem today in transplantation is the lack of suitable donor organs and tissues, the derivation of neural progenitor cells from hPSCs has opened a new avenue for regenerative medicine. In this review, we summarize the recent reports that show how to generate neural derivatives from hPSCs, and discuss the current evidence of using these cells in animal studies. We also highlight the possibilities and concerns of translating these hPSC-derived neurons for biomedical and clinical uses in order to fight against neurological disorders.
\end{abstract}

\section{Introduction}

Neurological disorders include a variety of hereditary and sporadic diseases that involve the chronic and progressive loss of neuronal structures and functions. We can divide these into two major groups depending on the onset of disease, which are (1) early onset neurodevelopment diseases and (2) late onset neurodegenerative diseases. Since aging is the most consistent risk factor for neurodegenerative diseases and we are now experiencing an increase in the numbers of the elderly population, it is of great importance to develop the treatments for these diseases. Although the rapid development of novel diagnostic methods and therapeutic approaches are in the stages of development, there is limited evidence of effective systems that can prevent and cure the diseases. Cell replacement therapy by using stem cells is a promising strategy to treat these diseases because certain pathological conditions are affected by neuronal loss. The possibility of generating abundant differentiated cells from human stem cells for cell replacement therapies provides a plausible avenue to treat such diseases. Neural stem cells that are isolated from adult donor or from fetal brain tissues have been considered as reasonable resources for this purpose; however, adult stem cells are of a very limited quantity, and histocompatibility is a major drawback.

Human pluripotent stem cell (hPSC) technologies, including human embryonic stem cells (hESCs) and induced pluripotent stem cells (iPSCs), are potentially prominent processes for manipulating human illnesses in terms of disease modeling, tissue engineering, drug discovery, and cell therapy. The pluripotent developmental potential of hPSCs and the success of transplanting their differentiated derivatives in animal disease models demonstrated the principle of using hPSC-derived cells as a regenerative source for transplantation therapies for human diseases. However, before hPSCs can be translated into clinical use, a thorough 
understanding of the basis of hPSCs is mandatory. One of the major concerns that hinders the application of hPSCs for cell and tissue therapy in human is histocompatibility. Notably, recent data support the concept that hESCs and their differentiated derivatives possess immune-privileged properties [1], suggesting that cells derived from hESCs may provide a potential tool for the induction of immunotolerance [2]. In another scenario, for which the term "personalized pluripotent cells" has been coined, people could use their own somatic cells to be reprogrammed back to the pluripotent cell state [3]. The feasibility of reprogramming was first demonstrated by somatic cell nuclear transfer (SCNT) or cloning [4]. Somatic cells of patients are fused with enucleated oocytes; thereafter, hESCs could be established in culture and be induced to in vitro differentiation to provide patientspecific cells and tissues [5]. However, the reprogramming of the somatic nucleus in an oocyte is still inefficient. In addition, accessing a source of human oocytes is not only a rare opportunity but it is also the ethical concern of the moment [6]. As an alternative to reprogramming by SCNT, adult human fibroblasts can be directly reprogrammed into a state that is similar to that of hESCs by the expression of only four factors: OCT4, KLF4, SOX2, and c-Myc [7] and such reprogrammed cells are termed as "induced pluripotent stem cells" or iPSCs.

Since hPSCs have great potential to differentiate into all cell types, the most common strategy in salvaging the neural function after injuries is not the direct transplantation of these pluripotent cells but applying their various differentiated cells instead. Specification of hPSCs in vitro along neural progenitor pathways would also allow the investigation of early human brain development, including regulatory signals for cell commitment and neurogenesis. Additionally, the cells could be used for the screening for new drugs and carcinogenic or toxic compounds that cannot be analyzed in vivo due to limited samples and ethical constraints. Ultimately, the potential utility of hPSC-derived neurons for treating neurological diseases is just at the beginning because there are several issues that need to be taken into account, that is, efficiency, safety, and functionality. In this paper, we review the promising benefits of hPSCs for the analysis and treatment of neurological disorders.

\section{Approaches for the Derivation of Neural Derivatives from Human Pluripotent Stem Cells}

The transplantation of stem cells which are committed to a specific lineage can avoid in vivo teratoma formation which is caused by the rapid growth and uncontrolled spontaneous differentiation of hPSCs [8]. Nevertheless, an efficient differentiation of hPSCs into clinically appropriate progenitors or specific targeting cell types remains a key challenge. The differentiation studies of hPSCs have intensively focused on exploring the roles of growth factors and small molecules [9-12]. Several approaches have been used to achieve in vitro neural differentiation from hPSCs, aimed at generating regionally specified neural progenitors or differentiated neurons/glial subtypes [13]. Reportedly, hPSCs have been differentiated successfully into several types of neural derivatives, including neural progenitor cells [11, 12, 14-20], neural crest progenitors [21], motor neurons [2225], sensory neurons [9], dopaminergic neurons [26, 27], and specific glial subtypes [28]. The differentiation systems were initially achieved through cell aggregation or embryoid body (EB) formation in the presence of retinoic acid (RA) as a starting point for the isolation and culture of highly purified populations of neural progenitor cells [16]. These progenitors were able to be cultivated for about 25 population doublings as neurospheres in suspension culture, and they express markers of the early neuroectoderm, such as Nestin, polysialylated (PSA) NCAM, Musashil, and PAX6 [16]. The neural progenitor cells were able to be differentiated into neurons, astrocytes, and to a minor degree of cells expressing oligodendrocyte markers. However, as hPSCs are pluripotent, the efficiency of neural conversion is limited and lineage selection is usually needed to ensure the enrichment of a specific differentiated population. Most of the lineage induction protocols employed the addition of morphogens or growth factors to the hESC aggregates in suspension cultures. For this reason, EB formation has some drawbacks. Because a high concentration of morphogens or growth factors is required in order for the factors to reach cells inside the aggregates [29], cells on the surface and those inside the aggregates will present a varied degree of exposure to morphogens, which create a wide range of cell lineages or cells at various developmental stages. In addition, it is impossible to visualize the continual change of cell morphology in response to treatment because of the cluster nature.

To overcome these drawbacks, a simpler way to reconstitute neural commitment in vitro and achieve efficient neural production relies upon monolayer differentiation of hPSCs. However, when applying a similar monolayer differentiation system used for directing mouse embryonic stem cells (mESCs) to neural lineage, hPSCs generated a large proportion of nonneural lineage cells. This is mainly due to the active BMP signaling pathway in hPSCs [30]. Therefore, the only approach that has been shown to induce efficient hESC neural differentiation is by directly inhibiting the BMP and/or SMAD signaling pathways $[12,31,32]$. Treatment of hPSCs with noggin, a BMP antagonist, generated a homogeneous, morphologically distinct population of cells that expressed neuroectodermal markers, including PAX6, Musashil, and SOX2 and with no detection of mesoderm and endoderm lineage markers [12]. Noggin alone appears to, at least, initiate hPSCs differentiation toward neural lineage. The formation of "neural rosettes" is another morphological marker of in vitro differentiation of hESCs to neural cells which is reminiscent of the in vivo structural formation of a developing neural tube [33]. The culture of hPSCs in chemically defined medium with noggin resulted in $\mathrm{PAX}^{+} / \mathrm{SOX}^{-}$neural rosettes, and the additional supplementation of fibroblast growth factor 2 (FGF2) induced $\mathrm{PAX}^{+} / \mathrm{SOX}^{+}$neural rosettes [34]. Rosetteforming neural stem cells expressing anterior markers of the nervous system, such as Forsel, have shown the broadest differentiation potential [35]. These cells propagated in the presence of FGF2 and retained Forsel expression, even though 
FGF2 was considered to caudalize the cell fate of neural stem cells [36]. Moreover, the cells in neural rosettes are capable of multiplying by symmetrical division over a period of time and are able to differentiate cell types of both anterior-posterior and central-peripheral types of the nervous system and could be maintained in a long-term culture by stimulating Sonic hedgehog (Shh) and Notch pathways [35]. Neural progenitor cells derived from hPSCs are also capable of producing astrocytes and oligodendrocytes, either under basal conditions or with the medium supplemented with ciliary neurotrophic factor or platelet-derived growth factor (PDGF) [37]. Mature astrocytes express specific astroglial markers such as glial fibrillary acidic protein (GFAP) and $S 100 \beta$. However, the proportion of oligodendrocytes differentiating from hPSCs among the differentiated progeny is generally very low [17]. It has been shown that Olig2 ${ }^{+}$neural progenitors can be readily differentiated from hESCs in response to SHH and RA [38]. These Olig $2^{+}$progenitors generate mostly motor neurons during the neurogenic period. Moreover, Olig2 $2^{+}$progenitors persist after neurogenesis and become oligodendrocytes. This suggests that the Olig $2^{+}$progenitors may differentiate into oligodendrocytes, and highlights the importance of Olig2 in oligodendrocyte development [39]. It is noted that during embryonic neurodevelopment, glial cells, for example, astrocytes and oligodendrocytes are generated after the birth of major neuronal types [40]. The same neurogenesis to gliogenesis transition is preserved when neuroectodermal cells are cultured [41] or hPSCs are differentiated along the neural lineage [42]. The noteworthy temporal sequence of neuronal and glial differentiation corresponds to the timeline observed from limited samples of fetal tissues [42].

It is suggested that the intrinsic program governing neuronal and glial lineage development is retrained in vitro and highlights the feasibility of obtaining specific neuronal subtypes from hPSCs when appropriate inducers are applied at an optimal time point. In addition to extrinsic factors, forced expression of specific transcription factors, for example, NGN2 and PAX6, could speed and enrich the generation of neural lineage from hPSCs [43-45]. However, this transcription factor-based approach is needed for the development of an exogenous DNA-free system prior to translating it to patients.

\section{Human Pluripotent Stem Cells Accelerate Biomedical Research for Neurological Disorders}

Understanding the molecular interactions underlying developmental disorders of the brain is hindered by limited accessibility to early embryos and an inadequate amount of stage- and cell type-specific materials. Although these developmental diseases are unlikely to be cured by cell replacement therapies, a complete picture of disease pathology by disease modeling will facilitate the discovery of effective compounds that can improve patient conditions [46]. Recent results indicate that the differentiation of hPSCs in culture follows the hierarchical set of signals that regulate embryonic development in the generation of the germ layers and specific cell types [47]. Establishment of in vitro differentiation models that recapitulate brain development will form the foundation for dissecting molecular interactions. The ability to access and manipulate populations representing early neural developmental stages in the hPSC differentiation cultures provides a new approach for addressing the questions of lineage commitment.

The investigation of neural induction paradigms in hPSCs has significant implications for the insights into early human brain development. In recent years, more sophisticated and chemically defined culture systems have been developed. Anti-BMP signaling is thought to play a crucial role in neural induction $[12,31]$. Further studies found that the high efficiency of neural induction with BMP antagonist treatment is consistent with its role in the default model of neural induction [48]. In addition to the shared signaling pathways, temporal consequences are similar between in vivo and in vitro systems. For example, during in vivo development, the neural tube formation completes when the human embryo is approximately 3 weeks old. On the other hand, in vitro differentiation of hPSCs toward neural lineage is characterized by the formation of a neural rosette observed at about days 15-17 of differentiation [12, 17], reminiscent of the transverse-section of the neural tube. It can be speculated that in vivo development events in terms of spatial and temporal changes are grossly recapitulated during the in vitro formation of neural rosettes. One of the characteristic features of neural progenitor cells is the positional identity they acquire during neural induction and patterning, which plays a key role in the fate specification of neuronal subtypes. The positional information is imparted upon neural progenitor cells via morphogenetic gradients secreted by surrounding tissues. Partially, to mimic the positional information in a culture petri dish, morphogens that affect rostrocaudal and dorsoventral fate choices are applied together or in sequence. Applying FGF8, which influences midhindbrain fate, and Shh, a ventralizing molecule, further induces hPSC-derived neuroepithelial cells into midbrain dopaminergic neurons [49]. On the other hand, the inhibition of Wnt signaling, together with the activation of SHH signaling, enhances forebrain induction from hPSCs [50]. Absence or presence of these positional morphogens in the in vitro differentiation system leads to the production of a variety of neuronal subtypes. This would mean that the addition of morphogens or small molecules at a specific time and space is needed to pattern neural progenitor cells into a particular neuronal subtype, and the foundation of this knowledge is originally based on the current understanding of neurodevelopment. The refinement of signaling pathways that control specific neuronal subtype specification in vitro will lay the foundation for studying affected neuron pathology in human neurological diseases.

In addition to modeling early brain development, iPSCs are a powerful tool for modeling diseases [46]. The idea of disease modeling is to derive iPSCs from the patient's own cells and then induce them into the pathogenic cell types in vitro. Several laboratories have already established hiPSCs from patients suffering from Parkinson's disease, amyotrophic lateral sclerosis, and spinal muscular dystrophy 
$[51,52]$. Upcoming sophisticated differentiation and purification protocols would be necessary to generate cells that show comparable physiological conditions to disease stages. Moreover, hPSCs and their differentiated derivatives could be applied to chemical compound screening assays for the development of new potential pharmaceuticals and toxic or mutagenic reagents [53]. While primary cell cultures or established cell lines are commonly used for both purposes, hPSCs offer several advantages. The developmental equivalence of hPSCderived and embryonic populations provide a more rigorous system for evaluating the teratogenic and embryotoxic effects of a substance, in addition to general mutagenic and cytotoxic effects [54]. A protocol based on the differentiation of hPSCs has been established and validated for use in toxicity testing [55]. Additionally, genetic modification enables the tailoring of hPSC lines for specific purposes. For example, specific genes can be altered to increase sensitivity to mutagens or drugs [56,57], or tissue-specific reporter genes can be introduced to detect changes in gene expression induced by toxic chemicals or therapeutic agents [58].

\section{Therapeutic Promises of Human Pluripotent Stem Cells for Neurological Disorders: Perspectives of Animal Studies}

Neurological disorders are the complex disintegration of neurons as well as many types of neuroglia in the brain and/or spinal cord. The early phase therapy, such as applying trypsin plasminogen activators (tPAs) in stroke patients during the first few hours, can be used only in early diagnosed patients [59]. Nevertheless, there is still no effective treatment, which can ameliorate that the functional deficit exists in the human subjects. Several researchers established the protocols to generate neural progenitor/stem cells, motor neurons, oligodendrocyte progenitor cells in vitro and then transplanted these cells into various animal models in order to verify the ability to restore neuronal functions in vivo. There are promising evidences of differentiation, maturation, and integration of the grafted cells into the endogenous neural circuitry in animal models $[60,61]$. The hPSC-derived cells, which were introduced into the animal models, were restricted to one specific cell lineage in order to reduce the risk of tumorigenesis when compared with the direct transplantation of hPSCs [62]. Experimental studies in animal models are necessary to extrapolate the therapeutic effects of transplanted cells. Currently, there are two main strategies for assessing the efficacy of hPSCs for treating neurological disorders in animal models. First, hPSC-derived neurons and/or neuroglia were substituted into mice with injured neural circuitry by using intracerebral transplantation. Second, hPSCs were delivered systemically or locally into the brain where they might act through some other mechanisms to promote the differentiation, such as immunomodulation, neuroprotection, and stimulation of angiogenesis.

The neural derivatives of hPSCs are plausible sources for cell replacement therapies. Several diseases were already experimented in an animal setting, such as stroke and brain ischemia [63-66], spinal cord injury [23, 67, 68], Parkinson's disease [69], spinal muscular atrophy, amyotrophic lateral sclerosis [24, 70], and demyelinating diseases [71]. These provisional studies were performed in specific diseases environment in order to endow a prospect of pathological conditions, in which the results could be implemented for clinical interpretations. In this section, we discuss some prominent examples of neurological disorders that have been conducted in animal transplantation studies using hPSCs.

4.1. Stroke. The vast majority of neurological disorders falls into the group of devastating pathology and takes place in the cerebral arteries which are called cerebrovascular accident (CVA) or stroke [72]. Stroke is an overwhelming condition with lifelong functional deficits in patients due to tremendous loss of neuronal circuitry in the brain. The recovery of stroke patients is often incomplete even when they have received physical therapy training to promote functional recovery. Consequently, there is great enthusiasm for using cell therapy to restore and replenish dead cells and tissues after brain injury an expectation of functional improvements. Stroke is typically a consequence of a thrombotic or embolic occlusion in a major cerebral artery, most often the middle cerebral artery (MCA). Experimental focal cerebral ischemia models have been established to imitate human stroke and serve as an indispensable tool in the field of stroke research [73]. Models of cerebral ischemia can either be artery or vein occlusion via mechanics or thromboembolisms. An ischemic model is categorized into global and focal ischemia. Global ischemia is the restriction of blood flow, affecting the entire brain area, whereas focal ischemia is characterized by a reduction of cerebral blood flow in a distinct region of the brain. The global ischemia can be further divided into complete and incomplete types, while focal ischemia can be performed in both focal and multifocal cerebral ischemia [74]. Moreover, cerebral vessel occlusion can occur either in the proximal middle cerebral artery (pMCAo) (large vessel occlusion) or distal MCA (dMCAo) (small vessel occlusion). Thrombotic occlusion can be induced either via the injection of blood clots or thrombin into the MCA or by photothrombosis after intravenous injection of Rose Bengal [75]. Recently, there has been evidence in hPSCs transplantation, which focused on cortical injury. The studies were performed by dMCAo rather than transient MCAo models, which turned to both cortical and striatal injury [63, 64]. These studies showed a substantially decreased infarct volume after the transplantation of neural progenitor cells derived from hPSCs. Nevertheless, until now, experimental stroke studies in transgenic animal models have had limited success. This highlights the significant contribution of vascular risk factors found in certain clinical situations, which is also viable in the human systems $[75,76]$.

4.2. Spinal Cord Injury. Spinal cord injury (SCI) is another important neurological disorder which can be used to reveal the therapeutic effects of transplanted cells in animal studies. SCI causes permanent paralysis in patients due to the low rate of regeneration in the central nervous system (CNS). Robust cell death in the injured region happens from seconds to weeks after SCI, which results in the formation of the 
cavities or cysts that block the ascending and descending neurotransmission. This phenomenon occurs immediately after SCI, including neuronal fiber damage, mass ischemic neural cell necrosis and apoptosis, and glial scar formation, and leads to extensive secondary tissue injuries. SCI is a devastating condition, characterized by the disruption of axonal connections, failure of axonal regeneration, and loss of motor and sensory function. The therapeutic promise of stem cells has been focused on cell replacement, but many obstacles remain, in particular neuronal integration following transplantation into the injured CNS. Various cell types have been selected based on their ability to form myelin protein, promote and guide axonal growth, and bridge the site of injury. In addition, transplanted cells also secrete trophic factors, which may have neuroprotective effects and/or promote plasticity in the spared spinal cord. Therefore, the advantageous effects of these cellular therapies are multifactorial and often difficult to attribute to one single mechanism. Most cell transplantations are delivered directly into the site of injury or adjacent area by injecting a few microliters of cell suspension via fine needles or glass capillaries. Attempts have been made to deliver cell substrates to the injured cord via intrathecal injection [77-79] or even systemically via intravenous infusions. Rodent models of SCI are used, and the transplantation is typically performed 1-2 weeks after the injury and referred to "subacute" treatment, since transplantations performed immediately after "acute" injury generally yield poor results due to the robust inflammatory response initiated at the time of injury [80]. In order to promote functional recovery, stem cell transplantation must be done after inflammatory responses. The optimal time-point for cell therapy contains several benefits, for instance, the inhibition of neuronal apoptosis and necrosis, the enhancement of neuronal regeneration, and the promotion of axon regeneration and remyelination; therefore, understanding the timeline of secondary damage cascades is definitely critical [81]. Reportedly, hPSCs have been investigated and their therapeutic efficacy and safety for SCI in vivo have been verified $[23,68]$. hESCs were differentiated into motor neuron progenitors (MPs) and oligodendrocyte progenitor cells (OPCs). The functional recovery was compared after transplanted either MPs or OPC alone, or the combination of MPs and OPCs into the SCI mice. The functional locomotor recovery of transplanted animals with MPs and OPCs showed significant improvement of the hind limb, better than the groups that were treated with a single cell type [23]. A protocol was recently developed for the creation of longterm self-renewing neuroepithelial-like stem (lt-NES) cells from hPSCs [68]. These hPSC-lt-NES cells exhibit reliable characteristics, including homogenous population, continuous expandability, stable neuronal/glial differentiation ability, and the capacity to generate functional mature neurons in a monolayer platform [68]. Promisingly, when transplanted into the SCI model (NOD-SCID mice), these transplanted cells have a comparable therapeutic potential, similar to neural stem cells (NSCs) derived from human fetal spinal cord (hsp-NSCs).
4.3. Parkinson's Disease. The key pathology of Parkinson's disease (PD) is motor symptoms. This is due to the progressive degeneration of mesencephalic dopaminergic (DA) neurons that project to the striatum and subsequent reductions in striatal dopamine levels. Initial pharmacological treatment with L-dihydroxyphenylalanine (L-DOPA) can ameliorate symptoms, but effectiveness of this compound gradually decreases overtime. The progression of motor deficits then requires additional treatments, including deep brain stimulation. The breakthrough lines of evidence of promising stem cell research are appealing as an alternative choice to fight against the disease. In the laboratory, PD animals can be induced by systemic injection of 1-methyl-4phenyl-1,2,3,6-tetrahydropyridine (MPTP) [69] or the central administration of the neurotoxin, 6-hydroxydopamine injection [82]. MPTP treatment is currently a gold standard for generating PD animal models. Ingestion of this compound results in the fabrication of motor deficits that are similar to PD. MPTP subsequently converts to $\mathrm{MPP}^{+}$ in the brain, which is later reuptaken into DA neurons by the dopamine transporter (DAT). $\mathrm{MPP}^{+}$exerts a blockage on the electron transport chain in the mitochondria of DA neurons and generates reactive oxygen species (ROS), which effectively kill the neurons [83]. There is still controversy as to whether MPTP-treated mice contained Lewy bodylike inclusions, a hallmark of PD. Only a few inclusions were found after 3 weeks of chronic MPTP treatment. By 24 weeks, several of the remaining tyrosine hydroxylase (TH) positive neurons contained $\alpha$-synuclein and ubiquitinimmunoreactive inclusions [84]. On the other hand, 6hydroxydopamine (6-OHDA) is preferentially transported into DA neurons by the DAT, where it gets accumulated and produces ROS. This toxin is needed for the administration via intraparenchymal injection because it does not cross the blood-brain barrier. Unilateral administration results in asymmetric circling behaviour, which is suitable for the evaluation of therapeutic interventions [85]. The preclinical study using MPTP-treated monkey models showed the therapeutic effects of transplanted cells at different stages, undifferentiated hESCs, and hESC-derived neural progenitor cells. As expected, the transplantation of undifferentiated hESCs at day 14 (before SHH and FGF8 induction) showed tumor formation. To induce neural progenitor cells for the transplantation, hESCs were induced with SHH for either 35 or 42 days prior to the addition of FGF8, BDNF, and GDNF for another 1-2 weeks. The grafts were well demarcated and showed no malignancy or even teratoma. The transplanted cells were mainly contributed to immature neural cells and DA neurons. The evaluation of functional neurological scores revealed that monkeys implanted with day 42 neurospheres had significant behavioural improvement over another group. The key statement of this finding was to support a prolonged differentiation maturation of neural progenitor cells that led to a favourable result regarding reduced tumor growth and functional grafts [69].

4.4. Motor Neuron Diseases. Recent progress in cell-based modeling using hPSC-derived motor neurons (MNs) has opened a new window to understanding the pathological 
development of motor neuron diseases. MNs exclusively reside within the ventral horn of the spinal cord and project axons to muscles to control their activity in organized and discrete patterns as the lowest unit in the hierarchy of the motor pathway. The most remarkable $\mathrm{MN}$ diseases are spinal muscular atrophy (SMA) and amyotrophic lateral sclerosis (ALS). SMA is characterized by severe muscle weakness, symmetrical proximal muscle weakness, lack of motor development, and hypotonia [86]. SMA is the cascade of genetic deficits, resulting in the decrease of survival of motor neuron (SMN) protein levels. The transgenic model of SMN gene deletion has yielded important insights into the pathogenesis of the disease. ALS, also known as Lou Gehrig's disease, is a devastating adult-onset neurodegenerative disorder, characterized by a progressive loss of both cortical and spinal motor neurons. The clinical manifestations of ALS are progressive myasthenia and general amyotrophy, eventually resulting in paralysis and death [87]. Transgenic mice that overexpress mutant human superoxide dismutase 1 (SOD1) gene reproduce clinical and histopathological features of human ALS. This animal model is of interest for the investigation of ALS pathogenesis and for the testing of therapeutic approaches [88]. Stem cell-based therapies have demonstrated therapeutic potential in SMA and ALS. For example, MNs derived from hiPSCs, which were obtained from an SMA patient, exhibited shortened neurite extensions and diminished survival in culture compared to healthy MNs [89]. Conversely, ALS patient-derived MNs did not present any defect [52]. MNs derived from hESCs proved their therapeutic capacity via in ovo and in vivo transplantation of the spinal cord [24]. The results revealed that MNs can survive for at least 6 weeks in the rat spinal cord and also outgrow the axons toward peripheral targets. This study provided strong promise for future applications in preclinical models and translational applications of hESC-derived MNs. It will be essential to develop animal models for specific conditions of $\mathrm{MN}$ diseases to address the question of whether hESCderived MNs can survive and function in a particular MN disease environment.

4.5. Multiple Sclerosis. Multiple sclerosis (MS) is an autoimmune-mediated inflammatory disease. It is characterized by multifocal regions of inflammation and myelin devastation, which leads to demyelination and neuronal loss. MS is presented by multiple signs and symptoms, with relapses and remissions of the disease stages. Even though there are several approved treatments for MS, many patients do not optimally respond to those approaches. A number of laboratory animals are described as demyelinating disease models. Experimental autoimmune encephalomyelitis (EAE) is one of the most commonly characterized disease and is employed as an animal model for MS [90]. EAE mice are a model of CNS autoimmune disease that follows immunization with certain CNS antigens and subsequent administration of heatinactivated Mycobacterium tuberculosis and pertussis toxin. Induced animals will develop a strong immune response with signs of inflammation, demyelination, axonal loss, and gliosis, which is similar to MS pathology in humans. Besides EAE, it is previously reported that an MS model could be induced by viral infection [71]. A number of human viral pathogens have been considered to be involved in eliciting myelinreactive lymphocytes and/or antibodies that subsequently infiltrate the CNS and damage the myelin sheath [91, 92]. Mouse hepatitis virus (MHV) infection results in an acute encephalomyelitis, followed by chronic demyelination in animals. This observation is similar to clinical and histologic profiles of MS patients. Human oligodendrocyte progenitor cells (OPCs) derived from hESCs transplanted in the MS model have been shown to promote remyelination in mice that are persistently infected with MHV [71]. The ability of preclinically applicable cells to facilitate remyelination in an animal model of MS would be a crucial step towards developing novel therapies.

\section{Safety Considerations of Using hPSC-Derived Neurons}

As mentioned, hPSCs offer a possible unlimited supply of disease-specific progenitor cells for regenerative medicine. The selected cell types can be variable, according to material sources, culture conditions, and differentiation protocols. These issues are important and need to be considered prior to translating preclinical outcomes into clinical studies. Undefined biological supplements, which are used for cell sustenance in the processes of isolation, expansion, and differentiation of hPSCs, may cause undesired problems in patients. For example, the maintenance of the hPSCs in growth-arrested mouse embryonic fibroblasts (MEFs) may worsen therapeutic potential due to the transmission of xenopathogens, altering the genetic background, and promoting the expression of immunogenic proteins in hPSCs [93]. Recently, there has been evidence of using xenofree iPSC-derived neural progenitor cells transplanted into ischemic stroke models [65]. This study represented the success of the derivation iPSCs in feeder- and serum-free systems. The cells could still be differentiated into functional neurons, which are transplantable into stroke animals.

Another challenge for the clinical considerations of hPSCs is how to efficiently induce and enrich pluripotent cells for a desired phenotype. In order to select desired cell types, certain approaches have been applied, such as antibodybased selection for specific surface antigens by FACS sorting. However, these approaches need to be improved in order to produce a large and viable population. To avoid tumor formation in the engrafted tissues, the pluripotent state of differentiated hPSCs needs to be verified to confirm no existing contamination of pluripotent cells among differentiated cells. Directing the pluripotent cells into the multipotent NSCs appears to be necessary for safety considerations and effectiveness in clinical translation. The precise stages of differentiation for transplantation remain unclear. The gene and epigenetic profiles are needed to validate reliable cell types before transplantation $[94,95]$. Therefore, safe and effective clinical translation of hPSCs for neurological disorders is required for thoroughly understanding of both inherent and noninherent cellular mechanisms that maintain pluripotency and differentiation programs. 


\section{Future Challenges of Neural Derivatives for Biomedical Research and Clinical Applications}

Based on the principle of developmental biology, a set of neurons and glia has been successfully differentiated from hPSCs. Modeling neurological diseases using hPSCs has the potential to provide a valuable impact on biomedical research and regenerative medicine. The possible risks are that the high variability in the protocols generates specific neuron subtypes and the ability to mimic disease-specific phenotypes. Manipulation of culture environment, such as oxidative stress, may enhance pathological phenotypes of neural derivatives derived from diseased iPSCs [96]. Although hPSC-derived neural derivatives were proved to be functional in vitro and were able to correct phenotypes of diseased mice, there are still several issues remaining to be solved prior to realizing clinical translation, for example, the purity of transplanting cells, sites of transplantation, graft versus host diseases, tumorigenesis, and integration of transplanted cells. The development of safer iPSCs is necessary to avoid exogenous DNA integration that can subsequently affect genomic alterations and cause tumor. Finding specific biomarkers for cell sorting may provide a solution to the selection of desired cell types for transplantation. In principle, patient-specific iPSCs should provide immunogenically matched tissues; however, further validation is still needed for safety issues in order to avoid any possible tissue rejections [97, 98].

Another key advantage of hiPSCs over the current transplantation approaches is the possibility of correcting mutations by homologous recombination technology. The generation of genetically corrected iPSCs by genome-editing technology is a mainstay for the advancement of iPSC technology to generate healthy iPSC lines for individual patients $[99,100]$. The differentiation of specific neural derivatives from genetically corrected iPSCs could provide a source of neurons for therapeutic transplantation. Besides, several neurological disorders are noncell autonomous, and neuronal death is driven by factors in the cellular environment, such as oxidative stress and inflammatory cytokines. The transplantation of nonneuronal cells, for example, astrocytes and oligodendrocytes, to refine the microenvironment conditions is thus a practical strategy. Another interesting concept in stem cell therapy is the dual effects of stem cell transplantation together with noninherent effects, such as rehabilitation or exercise. Since the majority of neurological disorder patients receive physiotherapy training, the combination of two approaches could enhance the therapeutic outcomes. To this end, the concerted efforts on hPSC research have made great progress toward cell replacement therapies; however, it is important to support the most carefully designed clinical studies for the best safety for patients in the future.

\section{Conflict of Interests}

The authors declare that they have no conflicts of interests.

\section{Authors' Contribution}

Nopporn Jongkamonwiwat and Parinya Noisa equally contributed to this work.

\section{Acknowledgments}

The authors apologize to those whose works are not cited because of space constraints. Parinya Noisa was supported by Suranaree University of Technology. Nopporn Jongkamonwiwat was supported by Thailand Research Fund (TRF), Srinakharinwirot University, and the Commission on Higher Education (CHE) for research funding (the Mid-Career University Faculty Program, Grant no. RSA5680003).

\section{References}

[1] L. Li, M. L. Baroja, A. Majumdar et al., "Human embryonic stem cells possess immune-privileged properties," Stem Cells, vol. 22, no. 4, pp. 4448-4456, 2004.

[2] P. Menendez, C. Bueno, L. Wang, and M. Bhatia, "Human embryonic stem cells: potential tool for achieving immunotolerance?" Stem Cell Reviews, vol. 1, no. 2, pp. 151-158, 2005.

[3] P. Noisa and R. Parnpai, "Technical challenges in the derivation of human pluripotent cells," Stem Cells International, vol. 2011, Article ID 907961, 7 pages, 2011.

[4] M. Tachibana, P. Amato, M. Sparman et al., "Human embryonic stem cells derived by somatic cell nuclear transfer," Cell, vol. 153, no. 6, pp. 61228-61238, 2013.

[5] A. J. French, S. H. Wood, and A. O. Trounson, "Human therapeutic cloning (NTSC): applying research from mammalian reproductive cloning," Stem Cell Reviews, vol. 2, no. 4, pp. 265276, 2006.

[6] E. Einsiedel, S. Premji, R. Geransar, N. C. Orton, T. Thavaratnam, and L. K. Bennett, "Diversity in public views toward stem cell sources and policies," Stem Cell Reviews and Reports, vol. 5, no. 2, pp. 102-107, 2009.

[7] K. Takahashi, K. Tanabe, M. Ohnuki et al., "Induction of pluripotent stem cells from adult human fibroblasts by defined factors," Cell, vol. 131, no. 5, pp. 861-872, 2007.

[8] A. Trounson, "Human embryonic stem cells: mother of all cell and tissue types," Reproductive Biomedicine Online, vol. 4, supplement 1, pp. 58-63, 2002.

[9] W. Chen, N. Jongkamonwiwat, L. Abbas et al., "Restoration of auditory evoked responses by human ES-cell-derived otic progenitors," Nature, vol. 490, no. 7419, pp. 278-282, 2012.

[10] P. Koch, T. Opitz, J. A. Steinbeck, J. Ladewig, and O. Brüstle, "A rosette-type, self-renewing human ES cell-derived neural stem cell with potential for in vitro instruction and synaptic integration," Proceedings of the National Academy of Sciences of the United States of America, vol. 106, no. 9, pp. 3225-3230, 2009.

[11] P. Itsykson, N. Ilouz, T. Turetsky et al., "Derivation of neural precursors from human embryonic stem cells in the presence of noggin," Molecular and Cellular Neuroscience, vol. 30, no. 1, pp. 24-36, 2005.

[12] L. Gerrard, L. Rodgers, and W. Cui, "Differentiation of human embryonic stem cells to neural lineages in adherent culture by blocking bone morphogenetic protein signaling," Stem Cells, vol. 23, no. 9, pp. 1234-1241, 2005. 
[13] S. Erceg, M. Ronaghi, and M. Stojković, "Human embryonic stem cell differentiation toward regional specific neural precursors," Stem Cells, vol. 27, no. 1, pp. 78-87, 2009.

[14] S. Narkilahti, K. Rajala, H. Pihlajamäki, R. Suuronen, O. Hovatta, and H. Skottman, "Monitoring and analysis of dynamic growth of human embryonic stem cells: comparison of automated instrumentation and conventional culturing methods," BioMedical Engineering Online, vol. 6, no. 11, 2007.

[15] B. E. Reubinoff, P. Itsykson, T. Turetsky et al., "Neural progenitors from human embryonic stem cells," Nature Biotechnology, vol. 19, no. 12, pp. 1134-1140, 2001.

[16] M. K. Carpenter, M. S. Inokuma, J. Denham, T. Mujtaba, C.P. Chiu, and M. S. Rao, "Enrichment of neurons and neural precursors from human embryonic stem cells," Experimental Neurology, vol. 172, no. 2, pp. 383-397, 2001.

[17] S. C. Zhang, M. Wernig, I. D. Duncan, O. Brüstle, and J. A. Thomson, "In vitro differentiation of transplantable neural precursors from human embryonic stem cells," Nature Biotechnology, vol. 19, no. 12, pp. 1129-1133, 2001.

[18] A. Trounson, "The production and directed differentiation of human embryonic stem cells," Endocrine Reviews, vol. 27, no. 2, pp. 208-219, 2006.

[19] R. Chaddah, M. Arntfield, S. Runciman et al., "Clonal neural stem cells from human embryonic stem cell colonies," The Journal of Neuroscience, vol. 32, no. 23, pp. 7771-7781, 2012.

[20] J. C. Pina-Crespo, M. Talantova, E. G. Cho et al., "Highfrequency hippocampal oscillations activated by optogenetic stimulation of transplanted human ESC-derived neurons," The Journal of Neuroscience, vol. 32, no. 45, pp. 15837-15842, 2012.

[21] G. Lee, H. Kim, Y. Elkabetz et al., "Isolation and directed differentiation of neural crest stem cells derived from human embryonic stem cells," Nature Biotechnology, vol. 25, no. 12, pp. 1468-1475, 2007.

[22] Y. Zhang, J. Wang, G. Chen, D. Fan, and M. Deng, "Inhibition of Sirtl promotes neural progenitors toward motoneuron differentiation from human embryonic stem cells," Biochemical and Biophysical Research Communications, vol. 404, no. 2, pp. 610614, 2011.

[23] S. Erceg, M. Ronaghi, M. Oria et al., “Transplanted oligodendrocytes and motoneuron progenitors generated from human embryonic stem cells promote locomotor recovery after spinal cord transection," Stem Cells, vol. 28, no. 9, pp. 1541-1549, 2010.

[24] H. Lee, G. A. Shamy, Y. Elkabetz et al., "Directed differentiation and transplantation of human embryonic stem cell-derived motoneurons," Stem Cells, vol. 25, no. 8, pp. 1931-1939, 2007.

[25] M. E. Hester, M. J. Murtha, S. Song et al., "Rapid and efficient generation of functional motor neurons from human pluripotent stem cells using gene delivered transcription factor codes," Molecular Therapy, vol. 19, no. 10, pp. 1905-1912, 2011.

[26] M. S. Cho, Y. E. Lee, J. Y. Kim et al., "Highly efficient and largescale generation of functional dopamine neurons from human embryonic stem cells," Proceedings of the National Academy of Sciences of the United States of America, vol. 105, no. 9, pp. 33923397, 2008.

[27] A. L. Perrier, V. Tabar, T. Barberi et al., "Derivation of midbrain dopamine neurons from human embryonic stem cells," Proceedings of the National Academy of Sciences of the United States of America, vol. 101, no. 34, pp. 12543-12548, 2004.

[28] R. Krencik, J. P. Weick, Y. Liu, Z.-J. Zhang, and S.-C. Zhang, "Specification of transplantable astroglial subtypes from human pluripotent stem cells," Nature Biotechnology, vol. 29, no. 6, pp. 528-534, 2011.
[29] H. Wichterle, I. Lieberam, J. A. Porter, and T. M. Jessell, "Directed differentiation of embryonic stem cells into motor neurons," Cell, vol. 110, no. 3, pp. 385-397, 2002.

[30] M. F. Pera, J. Andrade, S. Houssami et al., "Regulation of human embryonic stem cell differentiation by BMP-2 and its antagonist noggin," Journal of Cell Science, vol. 117, no. 7, pp. 1269-1280, 2004.

[31] S. M. Chambers, C. A. Fasano, E. P. Papapetrou, M. Tomishima, M. Sadelain, and L. Studer, "Highly efficient neural conversion of human ES and iPS cells by dual inhibition of SMAD signaling," Nature Biotechnology, vol. 27, no. 3, pp. 275-280, 2009.

[32] D. S. Kim, J. S. Lee, J. W. Leem et al., "Robust enhancement of neural differentiation from human ES and iPS cells regardless of their innate difference in differentiation propensity," Stem Cell Reviews and Reports, vol. 6, no. 2, pp. 270-281, 2010.

[33] R. O'Rahilly and F. Müller, "Neurulation in the normal human embryo," Ciba Foundation Symposium, vol. 181, pp. 70-82, 1994.

[34] S. Yao, S. Chen, J. Clark et al., "Long-term self-renewal and directed differentiation of human embryonic stem cells in chemically defined conditions," Proceedings of the National Academy of Sciences of the United States of America, vol. 103, no. 18, pp. 6907-6912, 2006.

[35] Y. Elkabetz, G. Panagiotakos, G. Al Shamy, N. D. Socci, V. Tabar, and L. Studer, "Human ES cell-derived neural rosettes reveal a functionally distinct early neural stem cell stage," Genes and Development, vol. 22, no. 2, pp. 152-165, 2008.

[36] S. C. Zhang, "Neural subtype specification from embryonic stem cells," Brain Pathology, vol. 16, no. 2, pp. 132-142, 2006.

[37] O. Brüstle, K. N. Jones, R. D. Learish et al., "Embryonic stem cell-derived glial precursors: a source of myelinating transplants," Science, vol. 285, no. 5428, pp. 754-756, 1999.

[38] X. J. Li, Z. W. Du, E. D. Zarnowska et al., "Specification of motoneurons from human embryonic stem cells," Nature Biotechnology, vol. 23, no. 2, pp. 215-221, 2005.

[39] Z. W. Du, X. J. Li, G. D. Nguyen, and S. C. Zhang, "Induced expression of Olig2 is sufficient for oligodendrocyte specification but not for motoneuron specification and astrocyte repression," Molecular and Cellular Neuroscience, vol. 33, no. 4, pp. 371-380, 2006.

[40] S. Temple, "The development of neural stem cells," Nature, vol. 414, no. 6859, pp. 112-117, 2001.

[41] X. Qian, Q. Shen, S. K. Goderie et al., "Timing of CNS cell generation: a programmed sequence of neuron and glial cell production from isolated murine cortical stem cells," Neuron, vol. 28, no. 1, pp. 69-80, 2000.

[42] J. Q. Wu, L. Habegger, P. Noisa et al., "Dynamic transcriptomes during neural differentiation of human embryonic stem cells revealed by short, long, and paired-end sequencing," Proceedings of the National Academy of Sciences of the United States of America, vol. 107, no. 11, pp. 5254-5259, 2010.

[43] Y. Zhang, C. Pak, Y. Han et al., "Rapid single-step induction of functional neurons from human pluripotent stem cells," Neuron, vol. 78, no. 5, pp. 785-798, 2013.

[44] E. C. Thoma, E. Wischmeyer, N. Offen et al., "Ectopic expression of neurogenin 2 alone is sufficient to induce differentiation of embryonic stem cells into mature neurons," PLoS ONE, vol. 7, no. 6, Article ID e38651, 2012.

[45] X. Zhang, C. T. Huang, J. Chen et al., "Pax6 is a human neuroectoderm cell fate determinant," Cell Stem Cell, vol. 7, no. 1, pp. 90-100, 2010. 
[46] T. Kunkanjanawan, P. Noisa, and R. Parnpai, "Modeling neurological disorders by human induced pluripotent stem cells," Journal of Biomedicine and Biotechnology, vol. 2011, Article ID 350131, 11 pages, 2011.

[47] M. Schuldiner, O. Yanuka, J. Itskovitz-Eldor, D. A. Melton, and N. Benvenisty, "Effects of eight growth factors on the differentiation of cells derived from human embryonic stem cells," Proceedings of the National Academy of Sciences of the United States of America, vol. 97, no. 21, pp. 11307-11312, 2000.

[48] D. C. Weinstein and A. Hemmati-Brivanlou, "Neural induction," Annual Review of Cell and Developmental Biology, vol. 15, pp. 411-433, 1999.

[49] S. H. Lee, N. Lumelsky, L. Studer, J. M. Auerbach, and R. D. McKay, "Efficient generation of midbrain and hindbrain neurons from mouse embryonic stem cells," Nature Biotechnology, vol. 18, no. 6, pp. 675-679, 2000.

[50] A. M. Maroof, S. Keros, J. A. Tyson et al., "Directed differentiation and functional maturation of cortical interneurons from human embryonic stem cells," Cell Stem Cell, vol. 12, no. 5, pp. 559-572, 2013.

[51] I. H. Park, N. Arora, H. Huo et al., "Disease-specific induced pluripotent stem cells," Cell, vol. 134, no. 5, pp. 877-886, 2008.

[52] J. T. Dimos, K. T. Rodolfa, K. K. Niakan et al., "Induced pluripotent stem cells generated from patients with ALS can be differentiated into motor neurons," Science, vol. 321, no. 5893, pp. 1218-1221, 2008.

[53] G. Lee, C. N. Ramirez, H. Kim et al., "Large-scale screening using familial dysautonomia induced pluripotent stem cells identifies compounds that rescue IKBKAP expression," Nature Biotechnology, vol. 30, no. 12, pp. 1244-1248, 2012.

[54] J. Rohwedel, K. Guan, C. Hegert, and A. M. Wobus, "Embryonic stem cells as an in vitro model for mutagenicity, cytotoxicity and embryotoxicity studies: present state and future prospects," Toxicology In Vitro, vol. 15, no. 6, pp. 741-753, 2001.

[55] S. Bremer, C. Pellizzer, S. Adler, M. Paparella, and J. De Lange, "Development of a testing strategy for detecting embryotoxic hazards of chemicals in vitro by using embryonic stem cell models," Alternatives to Laboratory Animals, vol. 30, supplement 2, pp. 107-109, 2002.

[56] A. Lorico, G. Rappa, R. A. Flavell, and A. C. Sartorelli, "Double knockout of the MRP gene leads to increased drug sensitivity in vitro," Cancer Research, vol. 56, no. 23, pp. 5351-5355, 1996.

[57] T. Ogi, Y. Shinkai, K. Tanaka, and H. Ohmori, "Pol $\kappa$ protects mammalian cells against the lethal and mutagenic effects of benzo[a]pyrene," Proceedings of the National Academy of Sciences of the United States of America, vol. 99, no. 24, pp. 15548-15553, 2002.

[58] K. Li, M. A. Ramirez, E. Rose, and A. L. Beaudet, "A gene fusion method to screen for regulatory effects on gene expression: application to the LDL receptor," Human Molecular Genetics, vol. 11, no. 26, pp. 3257-3265, 2002.

[59] W. M. Armstead, K. Ganguly, J. W. Kiessling et al., "Signaling, delivery and age as emerging issues in the benefit/risk ratio outcome of tPA for treatment of CNS ischemic disorders," Journal of Neurochemistry, vol. 113, no. 2, pp. 303-312, 2010.

[60] L. Ma, B. Hu, Y. Liu et al., "Human embryonic stem cell-derived GABA neurons correct locomotion deficits in quinolinic acidlesioned mice," Cell Stem Cell, vol. 10, no. 4, pp. 455-464, 2012.

[61] C. R. Nicholas, J. Chen, Y. Tang et al., "Functional maturation of hPSC-derived forebrain interneurons requires an extended timeline and mimics human neural development," Cell Stem Cell, vol. 12, no. 5, pp. 573-586, 2013.
[62] O. Tsuji, K. Miura, K. Fujiyoshi, S. Momoshima, M. Nakamura, and H. Okano, "Cell therapy for spinal cord injury by neural stem/progenitor cells derived from iPS/ES cells," Neurotherapeutics, vol. 8, no. 4, pp. 668-676, 2011.

[63] K. Jin, X. Mao, L. Xie et al., "Delayed transplantation of human neural precursor cells improves outcome from focal cerebral ischemia in aged rats," Aging Cell, vol. 9, no. 6, pp. 1076-1083, 2010.

[64] A. U. Hicks, R. S. Lappalainen, S. Narkilahti et al., “Transplantation of human embryonic stem cell-derived neural precursor cells and enriched environment after cortical stroke in rats: cell survival and functional recovery," European Journal of Neuroscience, vol. 29, no. 3, pp. 562-574, 2009.

[65] O. Mohamad, D. Drury-Stewart, M. Song et al., "Vector-free and transgene-free human iPS cells differentiate into functional neurons and enhance functional recovery after ischemic stroke in mice," PLoS ONE, vol. 8, no. 5, Article ID e64160, 2013.

[66] K. A. Puttonen, M. Ruponen, R. Kauppinen et al., "Improved method of producing human neural progenitor cells of high purity and in large quantities from pluripotent stem cells for transplantation studies," Cell Transplant, 2012.

[67] M. Sundberg, P.-H. Andersson, E. A. kesson et al., "Markers of pluripotency and differentiation in human neural precursor cells derived from embryonic stem cells and CNS tissue," Cell Transplantation, vol. 20, no. 2, pp. 177-191, 2011.

[68] Y. Fujimoto, M. Abematsu, A. Falk et al., "Treatment of a mouse model of spinal cord injury by transplantation of human induced pluripotent stem cell-derived long-term self-renewing neuroepithelial-like," Stem Cells, vol. 30, no. 6, pp. 1163-1173, 2012.

[69] D. Doi, A. Morizane, T. Kikuchi et al., "Prolonged maturation culture favors a reduction in the tumorigenicity and the dopaminergic function of human ESC-derived neural cells in a primate model of Parkinson's disease," Stem Cells, vol. 30, no. 5, pp. 935-945, 2012.

[70] Y. Luo, Y. Fan, X. Chen et al., "Generation of induced pluripotent stem cells from Asian patients with chronic neurodegenerative diseases," Journal of Reproduction and Development, vol. 58, no. 5, pp. 515-521, 2012.

[71] E. Tirotta, K. S. Carbajal, C. S. Schaumburg, L. Whitman, and T. E. Lane, "Cell replacement therapies to promote remyelination in a viral model of demyelination," Journal of Neuroimmunology, vol. 224, no. 1-2, pp. 101-107, 2010.

[72] WHO, Neurological Disorders : Public Health Challenges, World Health Organization, Geneva, Switzerland, 2006.

[73] A. Durukan and T. Tatlisumak, "Acute ischemic stroke: overview of major experimental rodent models, pathophysiology, and therapy of focal cerebral ischemia," Pharmacology Biochemistry and Behavior, vol. 87, no. 1, pp. 179-197, 2007.

[74] S. M. Graham, L. D. McCullough, and S. J. Murphy, "Animal models of ischemic stroke: balancing experimental aims and animal care," Comparative Medicine, vol. 54, no. 5, pp. 486-496, 2004.

[75] M. Bacigaluppi, G. Comi, and D. M. Hermann, "Animal models of ischemic stroke-part two: modeling cerebral ischemia," The Open Neurology Journal, vol. 4, pp. 34-38, 2010.

[76] M. Philip, M. Benatar, M. Fisher, and S. I. Savitz, "Methodological quality of animal studies of neuroprotective agents currently in phase II/III acute ischemic stroke trials," Stroke, vol. 40, no. 2, pp. 577-581, 2009.

[77] R. E. White, M. Rao, J. C. Gensel, D. M. McTigue, B. K. Kaspar, and L. B. Jakeman, "Transforming growth factor $\alpha$ transforms 
astrocytes to a growth-supportive phenotype after spinal cord injury," The Journal of Neuroscience, vol. 31, no. 42, pp. 1517315187, 2011.

[78] J. F. Bonner, A. Blesch, B. Neuhuber, and I. Fischer, "Promoting directional axon growth from neural progenitors grafted into the injured spinal cord," Journal of Neuroscience Research, vol. 88, no. 6, pp. 1182-1192, 2010.

[79] G. W. Hawryluk, A. Mothe, J. Wang et al., "An in vivo characterization of trophic factor production following neural precursor cell or bone marrow stromal cell transplantation for spinal cord injury," Stem Cells and Development, vol. 21, no. 12, pp. 2222-2238, 2012.

[80] W. Tetzlaff, E. B. Okon, S. Karimi-Abdolrezaee et al., "A systematic review of cellular transplantation therapies for spinal cord injury," Journal of Neurotrauma, vol. 28, no. 8, pp. 16111682, 2011.

[81] D. Garbossa, M. Boido, M. Fontanella, C. Fronda, A. Ducati, and A. Vercelli, "Recent therapeutic strategies for spinal cord injury treatment: possible role of stem cells," Neurosurgical Review, vol. 35, no. 3, pp. 293-311, 2012.

[82] H. J. Im, W. Hwang do, H. K. Lee et al., "In vivo visualization and monitoring of viable neural stem cells using noninvasive bioluminescence imaging in the 6-hydroxydopamine-induced mouse model of Parkinson disease," Molecular Imaging, vol. 12, no. 4, pp. 224-234, 2013.

[83] W. Dauer and S. Przedborski, "Parkinson's disease: mechanisms and models," Neuron, vol. 39, no. 6, pp. 889-909, 2003.

[84] G. E. Meredith, S. Totterdell, E. Petroske, K. Santa Cruz, R. C. Callison Jr., and Y.-S. Lau, "Lysosomal malfunction accompanies alpha-synuclein aggregation in a progressive mouse model of Parkinson's disease," Brain Research, vol. 956, no. 1, pp. 156165, 2002.

[85] S. Duty and P. Jenner, "Animal models of Parkinson's disease: a source of novel treatments and clues to the cause of the disease," British Journal of Pharmacology, vol. 164, no. 4, pp. 1357-1391, 2011.

[86] E. Zanoteli, J. R. Maximino, U. Conti Reed, and G. Chadi, "Spinal muscular atrophy: from animal model to clinical trial," Functional Neurology, vol. 25, no. 2, pp. 73-79, 2010.

[87] X. Li, Y. Guan, Y. Chen et al., "Expression of Wnt5a and its receptor Fzd2 is changed in the spinal cord of adult amyotrophic lateral sclerosis transgenic mice," International Journal of Clinical and Experimental Pathology, vol. 6, no. 7, pp. 1245-1260, 2013.

[88] S. Marconi, M. Bonaconsa, I. Scambi et al., "Systemic treatment with adipose-derived mesenchymal stem cells ameliorates clinical and pathological features in the amyotrophic lateral sclerosis murine model," Neuroscience, vol. 28, no. 248C, pp. 333-343, 2013.

[89] A. D. Ebert, J. Yu, F. F. Rose Jr. et al., "Induced pluripotent stem cells from a spinal muscular atrophy patient," Nature, vol. 457, no. 7227, pp. 277-280, 2009.

[90] L. Bai, J. Hecker, A. Kerstetter et al., "Myelin repair and functional recovery mediated by neural cell transplantation in a mouse model of multiple sclerosis," Neuroscience Bulletin, vol. 29, no. 2, pp. 239-250, 2013.

[91] A. Ascherio and K. L. Munger, "Environmental risk factors for multiple sclerosis-part I: the role of infection," Annals of Neurology, vol. 61, no. 4, pp. 288-299, 2007.

[92] A. Ascherio and K. L. Munger, "Environmental risk factors for multiple sclerosis-part II: noninfectious factors," Annals of Neurology, vol. 61, no. 6, pp. 504-513, 2007.
[93] M. J. Martin, A. Muotri, F. Gage, and A. Varki, "Human embryonic stem cells express an immunogenic nonhuman sialic acid," Nature Medicine, vol. 11, no. 2, pp. 228-232, 2005.

[94] B. E. Bernstein, T. S. Mikkelsen, X. Xie et al., "A bivalent chromatin structure marks key developmental genes in embryonic stem cells," Cell, vol. 125, no. 2, pp. 315-326, 2006.

[95] T. S. Mikkelsen, M. Ku, D. B. Jaffe et al., "Genome-wide maps of chromatin state in pluripotent and lineage-committed cells," Nature, vol. 448, no. 7153, pp. 553-560, 2007.

[96] H. N. Nguyen, B. Byers, B. Cord et al., "LRRK2 mutant iPSCderived DA neurons demonstrate increased susceptibility to oxidative stress," Cell Stem Cell, vol. 8, no. 3, pp. 267-280, 2011.

[97] T. Zhao, Z. N. Zhang, Z. Rong, and Y. Xu, "Immunogenicity of induced pluripotent stem cells," Nature, vol. 474, no. 7350, pp. 212-215, 2011.

[98] R. Araki, M. Uda, Y. Hoki et al., "Negligible immunogenicity of terminally differentiated cells derived from induced pluripotent or embryonic stem cells," Nature, vol. 494, no. 7435, pp. 100-104, 2013.

[99] F. Soldner, J. Laganière, A. W. Cheng et al., "Generation of isogenic pluripotent stem cells differing exclusively at two early onset parkinson point mutations," Cell, vol. 146, no. 2, pp. 318331, 2011.

[100] S. Corti, M. Nizzardo, C. Simone et al., "Genetic correction of human induced pluripotent stem cells from patients with spinal muscular atrophy," Science Translational Medicine, vol. 4, no. 165, p. 165ra162, 2012. 

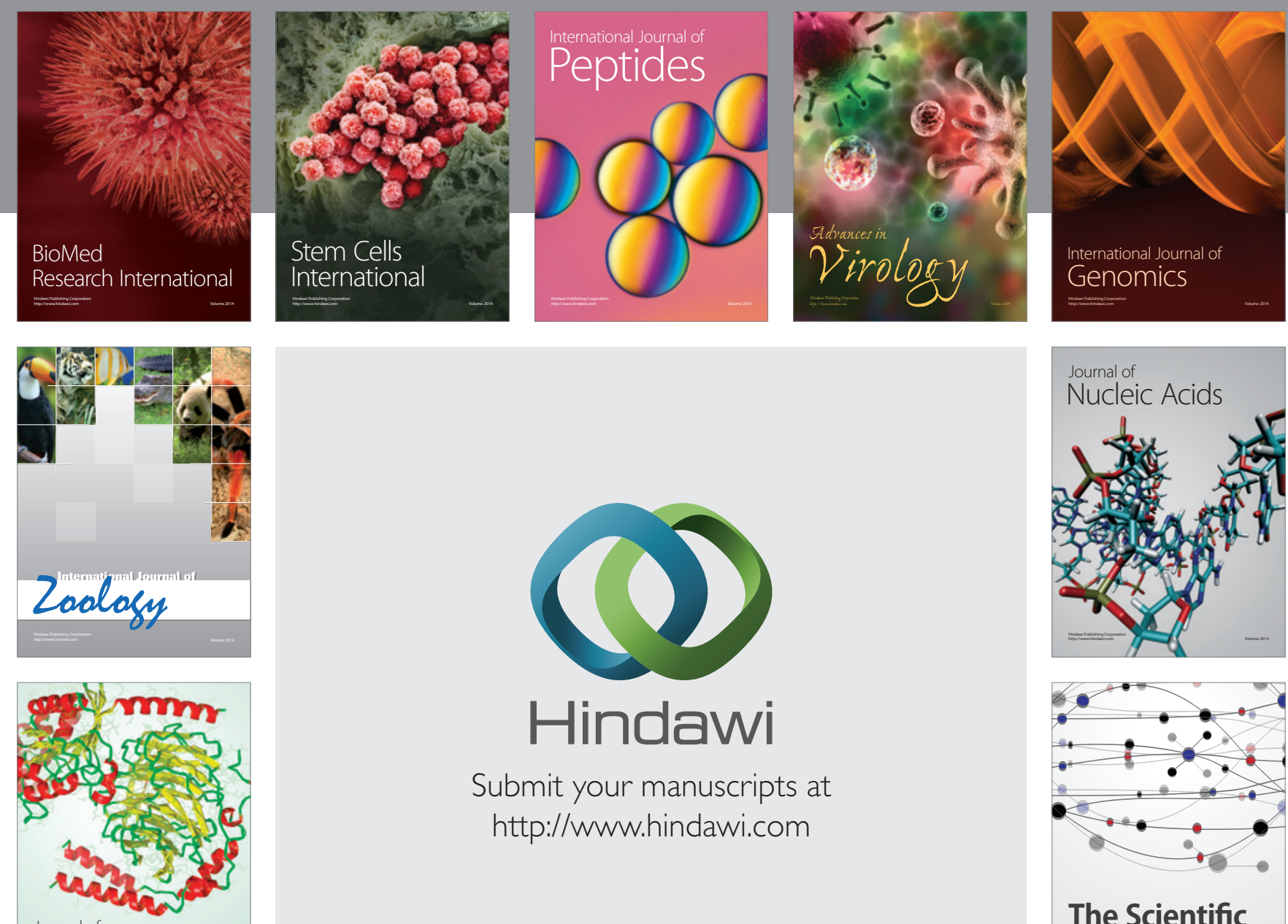

Submit your manuscripts at

http://www.hindawi.com

Journal of
Signal Transduction
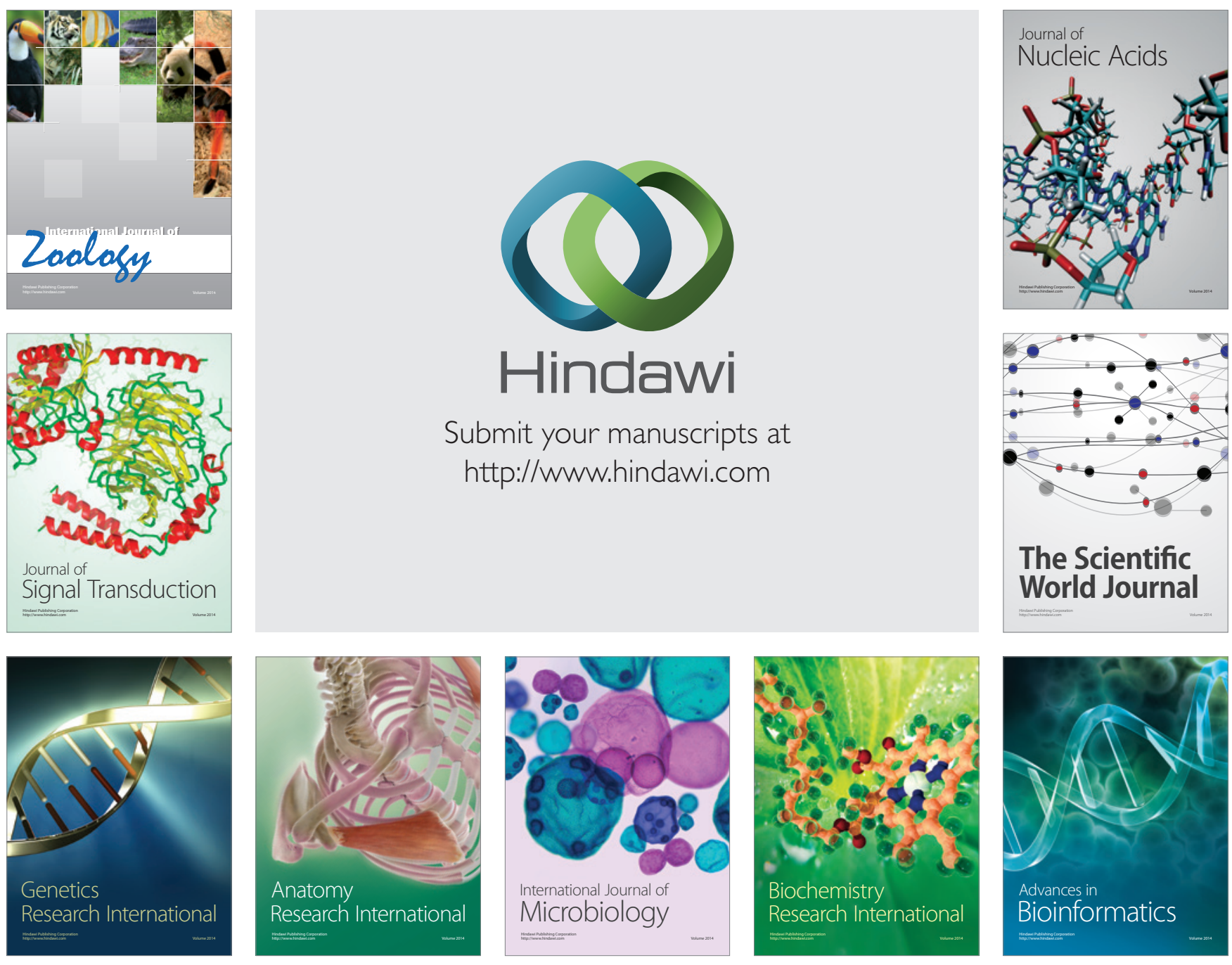

The Scientific World Journal
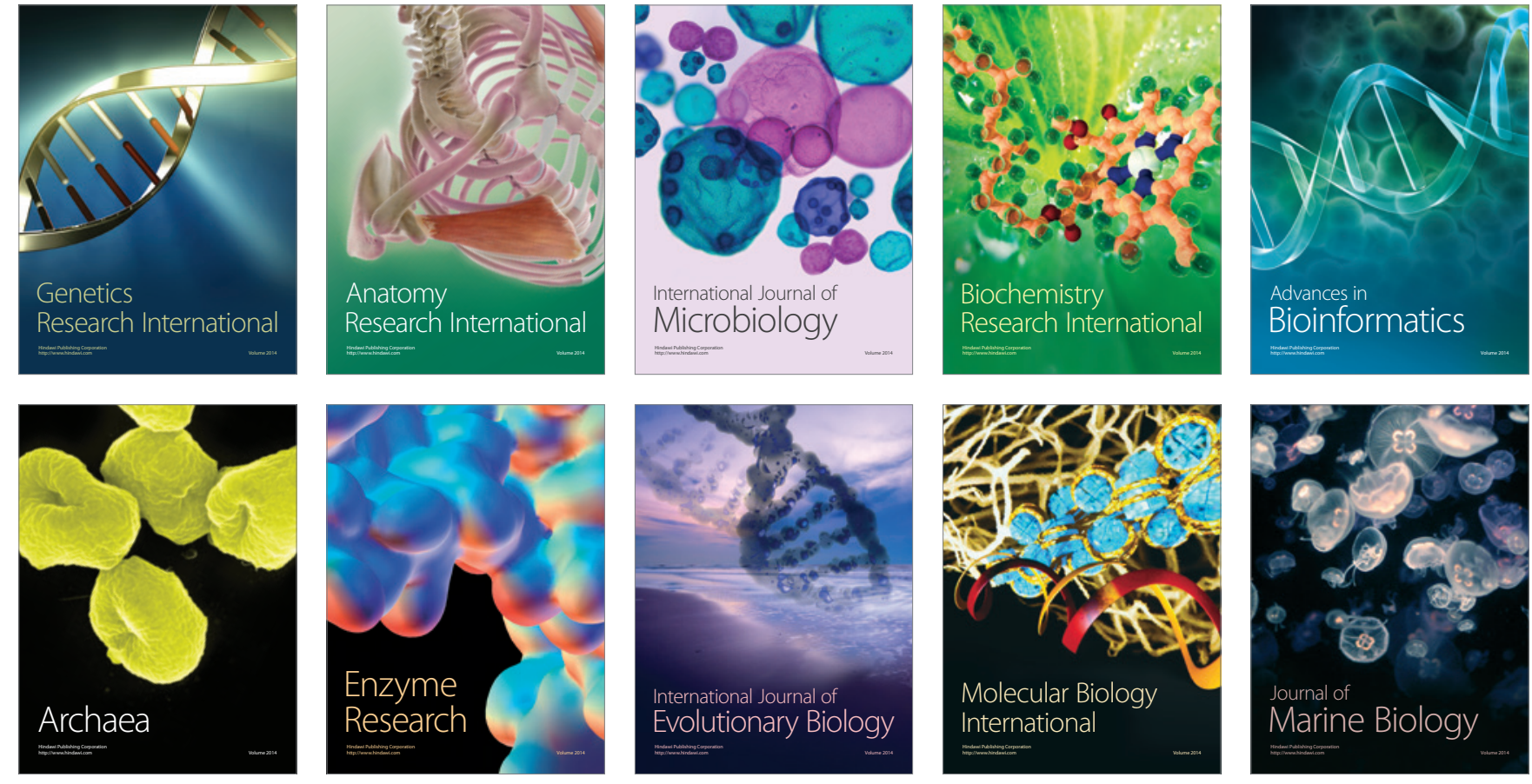